\section{E-021 PATHOPHYSIOLOGY, STROKE TYPES, COLLATERAL FORMATION AND ANEURYSMS IN MOYAMOYA DISEASE/MOYA MOYA SYNDROME}

S Ahmad*. Diagnostic and Interventional Neuroradiology Department, Lahore General Hospital, Lahore, Pakistan

\subsection{6/neurintsurg-2021-SNIS.117}

Objective Moyamoya disease (MMD) is a rare condition where the supraclinoid part of internal carotid artery is slowly and progressively becoming stenotic. The moyamoya syndrome (MMS) is similar to MMD but might be associated with Down's syndrome, neurofibromatosis type 1 (NF-1), prior irradiation, and sickle-cell disease. Phenotypic differences between moyamoya disease (MMD) and moyamoya syndrome (MMS) remain unclear. The prevalence of aneurysm formation in moyamoya disease (MMD) is approximately $14 \%$ in adults and rare in children, and it represents a major potential hemorrhagic risk. In this study, we will explore clinical and angiographic features of $\mathrm{MMD} / \mathrm{MMS}$, presentation, classification, association with aneurysms.
Methods This was a retrospective cross-sectional study. Imaging studies and clinical records of 13 patients who were admitted to our hospital between January 2019 and December 2020 and were diagnosed with MMD/MMS were reviewed. Angiographic findings and clinical features of MMD and associated aneurysms were reviewed. Collateral vessels were classified into three subgroups.

Results The study consisted of 13 patients, 38.5\% female, $61.5 \%$ males with a bimodal age distribution. 8 cases had bilateral involvement while unilateral MMD cases were only 5. All 38.5\% patients with aneurysms presented with rupture, and have unilateral MMD with only single case of bilateral MMD and aneurysm. The commonest location of aneurysm was distal basilar artery i-e 3 cases, rest of 2 cases had AcomA aneurysm. In one case of MMS, there was a basilar tip aneurysm with underlying vasculitis. Posterior circulation was involved in $46.1 \%$ cases. In $76.9 \%$ cases extensive collateral formation in lenticulostriate, choroidal and thalamic territories were evident.

Conclusions MMD is presented most commonly with ischemic stroke in the pediatric age group and with hemorrhagic stroke

\begin{tabular}{|c|c|c|c|c|c|}
\hline & $\begin{array}{l}\text { Initial Clinical } \\
\text { Menifestation }\end{array}$ & Type Of Stroke & Angiographic Characteristics & $\begin{array}{l}\text { Association with } \\
\text { Aneurysms }\end{array}$ & $\begin{array}{l}\text { Type of Collaterals ( } \\
\text { lenticulostriates/Thalamicl } \\
\text { Choroidal }\end{array}$ \\
\hline \multicolumn{6}{|c|}{ Age/Sex } \\
\hline $20 y / M$ & $\begin{array}{l}\text { Headache followed by } \\
\text { sudden loss of } \\
\text { consciousness }\end{array}$ & Hemorrhagic stroke/ICH & $\begin{array}{l}\text { B/L MMD -Bilateral ICA stenosis at } \\
\text { supraclinoid segment involving both ACAs \& } \\
\text { MCAs } \\
\text { Posterior circulation was involved } \\
\text { Incidental finding of intradural duplication of } \\
\text { VA }\end{array}$ & No & $\begin{array}{l}\text { Only thalamic collateral anastomosis } \\
\text { evident supplying bilateral cortex }\end{array}$ \\
\hline $38 \mathrm{y} / \mathrm{F}$ & Presented with ictus & Hemorrhagic stroke/ICH & $\begin{array}{l}\text { B/L MMD-Bilateral ICA stenosis at supraclinoid } \\
\text { region,bilateral ACAs \& MCAs involved } \\
\text { Transdural angiogenesis, posterior circulation } \\
\text { not involved }\end{array}$ & No & $\begin{array}{l}\text { Choroidal anastomosis and } \\
\text { lenticulostriate anastomosis evident }\end{array}$ \\
\hline \multirow[t]{2}{*}{ 30y/M } & $\begin{array}{l}\text { Presented with headache } \\
\text { and weakness of left }\end{array}$ & $\begin{array}{l}\text { Ischemic stroke/Right } \\
\text { ACA territory }\end{array}$ & $\begin{array}{l}\text { B/L MMD- Bilatteral ICA stenosis at } \\
\text { supraclinoid region involving both ACAs and } \\
\text { MCAs }\end{array}$ & No & $\begin{array}{l}\text { Thalamic perforator collaterals } \\
\text { anastomosis supplying the whole } \\
\text { cortex }\end{array}$ \\
\hline & lower limb & & $\begin{array}{l}\text { Transdural angiogenesis, posterior circulation } \\
\text { involved }\end{array}$ & & \\
\hline \multirow[t]{3}{*}{$45 y / M$} & $\begin{array}{l}\text { Presented with headache } \\
\text { followed by loss of }\end{array}$ & $\begin{array}{l}\text { Hemorrhagic stroke/ } \\
\text { SAH }\end{array}$ & $\begin{array}{l}\text { Unilateral MMD- Left ICA stenosis at } \\
\text { supraclinoid region, both sided ACA\& MCA } \\
\text { involved }\end{array}$ & Basilar tip aneurysm, left & $\begin{array}{l}\text { No lenticulostriates or choroidal } \\
\text { collaterals,only thalamic collaterals } \\
\text { showed }\end{array}$ \\
\hline & consciousness & & $\begin{array}{l}\text { transdural angiogenesis, Right ICA stenosis } \\
\text { proximal part }\end{array}$ & PCA hypoplastic, right PCA & $\begin{array}{l}\text { anastomosis supplying the whole } \\
\text { cerebral hemisphere }\end{array}$ \\
\hline & & & & $\begin{array}{l}\text { dilated and supplying the } \\
\text { whole cortex }\end{array}$ & \\
\hline $49 y / M$ & $\begin{array}{l}\text { Presented with sudden } \\
\text { headache and loss of }\end{array}$ & $\begin{array}{l}\text { Hemorrhagic stroke/ } \\
\text { SAH }\end{array}$ & $\begin{array}{l}\text { Unilateral MMD-Right ICA stenosis at } \\
\text { supraclinoid region, Right ECA supplying the } \\
\text { cortex }\end{array}$ & Large AcomA aneurysm & No collaterals evident \\
\hline & consciousness & & $\begin{array}{l}\text { through transdural angiogenesis, Left ICA } \\
\text { supplying the right ACA and MCA through } \\
\text { AComA, posterior circulation not involved }\end{array}$ & filling from left $A C A$ & \\
\hline $17 y / M$ & $\begin{array}{l}\text { Presented with right sided } \\
\text { hemiplegia/old left CVA }\end{array}$ & $\begin{array}{l}\text { Ischemic stroke/ } \\
\text { Bilateral CVA }\end{array}$ & $\begin{array}{l}\text { Bilateral MMD-Bilateral ICA stenosis at } \\
\text { supraclinoid region,both ACAs and MCAs } \\
\text { involved } \\
\text { Transdural angiogenesis, posterior circulation } \\
\text { involved }\end{array}$ & No & $\begin{array}{l}\text { Only thalamic collateral anastomosis } \\
\text { evident }\end{array}$ \\
\hline
\end{tabular}




\begin{tabular}{|c|c|c|c|c|c|}
\hline $15 y / F$ & Presented with acute ictus & $\begin{array}{l}\text { Hemorrhagic strokel } \\
\text { SAH }\end{array}$ & $\begin{array}{l}\text { Unilateral MMD -Left ICA stenosis at } \\
\text { supraclinoid region,left ACA involved, posterior } \\
\text { circulation not involved, Right ICA supplying } \\
\text { the left MCA through AcomA }\end{array}$ & $\begin{array}{l}\text { AComA aneurysm filling } \\
\text { from } \\
\text { right ACA }\end{array}$ & No collaterals evident \\
\hline 35y/M & $\begin{array}{l}\text { Headache followed by loss } \\
\text { of consciousness }\end{array}$ & Hemorrhagic stroke/IVH & $\begin{array}{l}\text { Bilateral MMD-Bilateral ICA stenosis at } \\
\text { supraclinoid region,ACA,MCA intact } \\
\text { posterior circulation involved }\end{array}$ & No & $\begin{array}{l}\text { Lenticulostriate, choroidal and } \\
\text { thalamic collateral formation with } \\
\text { anastomosis } \\
\text { seen }\end{array}$ \\
\hline $8 y / F$ & $\begin{array}{l}\text { Presented with sudden } \\
\text { bilateral lower limb } \\
\text { weakness, fits, headache }\end{array}$ & $\begin{array}{l}\text { Ischemic stroke/B/L } \\
\text { ACA territory infarcts }\end{array}$ & $\begin{array}{l}\text { Bilateral MMD-Bilateral ICA stenosis at } \\
\text { supraclinoid region, posterior circulation }\end{array}$ & No & $\begin{array}{l}\text { Lenticulostriates, choroidal and } \\
\text { thalamic collateral anastomosis } \\
\text { evident }\end{array}$ \\
\hline 43y/M & $\begin{array}{l}\text { Presented with headache } \\
\text { followed by loss of } \\
\text { consciousness }\end{array}$ & $\begin{array}{l}\text { Hemorrhagic stroke/ } \\
\text { SAH }\end{array}$ & $\begin{array}{l}\text { Bilateral MMD- Bilateral ICA stenosis at } \\
\text { supraclnoid region,posterior circulation } \\
\text { involved }\end{array}$ & Basilar tip aneurysm & $\begin{array}{l}\text { Thalamic collaterals anastomosis } \\
\text { supplying the whole cortex }\end{array}$ \\
\hline $18 y / F$ & $\begin{array}{l}\text { Presented with headache } \\
\text { followed by loss of } \\
\text { consciousness }\end{array}$ & Hemorrhagic stroke/ICH & $\begin{array}{l}\text { Unilateral MMD- Left ICA stenosis at } \\
\text { supraclinoid region, posterior circulation not } \\
\text { involved, posterior choroidal artery and } \\
\text { anterior choroidal artery showed anastmosis }\end{array}$ & No & $\begin{array}{l}\text { Lenticulostriate collateral anastomosis } \\
\text { seen }\end{array}$ \\
\hline $3 y / F$ & $\begin{array}{l}\text { Presented with left sided } \\
\text { weakness }\end{array}$ & $\begin{array}{l}\text { Ischemic stroke/Right } \\
\text { sided infarct }\end{array}$ & $\begin{array}{l}\text { Bilateral MMD- Bilateral ICA stenosis at } \\
\text { supraclinoid region, ACA \& MCA not involved } \\
\text { posterior circulation not involved }\end{array}$ & No & $\begin{array}{l}\text { Extensive lenticulostriate and } \\
\text { choroidal collateral formation and } \\
\text { anastomosis seen }\end{array}$ \\
\hline $12 \mathrm{y} / \mathrm{M}$ & $\begin{array}{l}\text { Presented with fever, neck } \\
\text { rigitidy, left sided weakness }\end{array}$ & $\begin{array}{l}\text { Hemorrhagic stroke/ } \\
\text { SAH }\end{array}$ & $\begin{array}{l}\text { Unilateral MMD - Left ICA stenosis at } \\
\text { supraclinoid region, posterior circulation not } \\
\text { involved }\end{array}$ & Distal basilar tip aneurysm & No collaterals evident \\
\hline
\end{tabular}

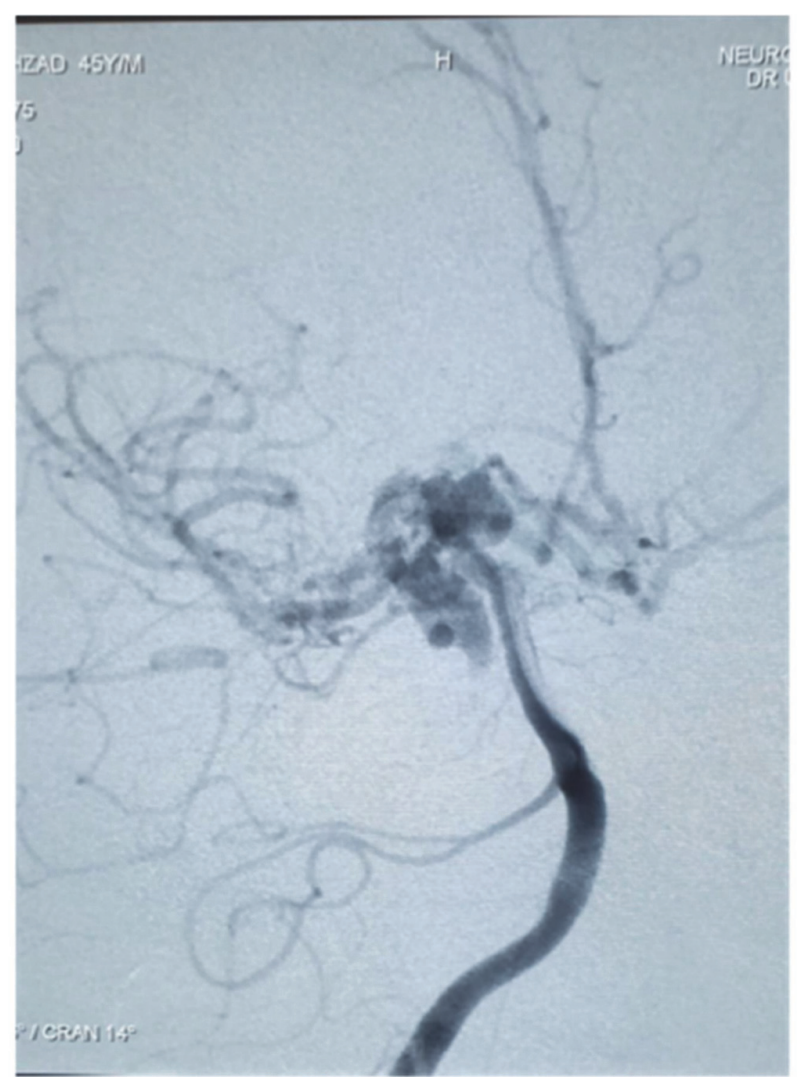

\section{Abstract E-021 Figure 1}

in the adult age group. MMD-associated intracranial aneurysms frequently occur in patients presenting with hemorrhagic unilateral MMD and are associated with an extremely high rate of rupture. Long-standing hemodynamic stress might contribute to the formation of an aneurysm.

Disclosures S. Ahmad: None.

\section{E-022 VIZ RECRUIT ICH VOLUME INCREASED THE SCREENING RATE AND ENROLLMENT IN ENRICH}

${ }^{1}$ B Jankowitz ${ }^{*},{ }^{2}$ J Davies, ${ }^{3} \mathrm{C}$ Schirmer, ${ }^{4} \mathrm{~J}$ Day, ${ }^{5} \mathrm{O}$ Bibas, ${ }^{6} \mathrm{~N}$ Levkovitz, ${ }^{7}$ E Blanc, ${ }^{8} \mathrm{G}$ Pradilla. ${ }^{1}$ Neurosurgery, Cooper Neurological Institute, Camden, Nj; ${ }^{2}$ Neurosurgery, UBNS, Buffalo, NY; ${ }^{3}$ Neurosurgery, Geisinger, Wilkes Barre, PA; ${ }^{4}$ Neurosurgery, UAMS, LIttle Rock, AR: ${ }^{5}$ Data Analytics, Viz.ai, Israel, Israel; ${ }^{6}$ Product Development, Viz.ai, Israel, Israel; ${ }^{7}$ Viz.ai, Israel, Israel; ${ }^{8}$ Neurosurgery, Emory, Atlanta, GA

\subsection{6/neurintsurg-2021-SNIS.118}

Introduction Early Minimally invasive Removal of IntraCerebral Hemorrhage (ENRICH) is a RCT evaluating the efficacy of minimally invasive surgery (MIS) for ICH. AI ENRICH is a prospective trial operating within and in parallel to the ENRICH trial that utilizes an AI application, Viz RECRUIT ICH Volume, to identify and segment ICH to quickly identify potentially eligible subjects.

Methods Non-contrast CT scans performed at 3 participating US hospitals were evaluated specifically for a parenchymal hemorrhage by Viz RECRUIT ICH Volume. Participating health care professionals downloaded a phone application that allowed users to be notified for any hemorrhage $\geq 5 \mathrm{~mL}$. Screening and enrollment rates are based on participating hospitals reports.

Results Over a combined period of 374 days, 20,128 CT scans were evaluated by the application, yielding a total of 110 patients that met ENRICH inclusion criteria for hematoma volume $(30-80 \mathrm{~mL})$. Of these, 9 patients were enrolled. The 